\title{
On the Frequency Domain Relaxation Processes in Gallium Doped CdTe and $\mathrm{Cd}_{0.99} \mathrm{Mn}_{0.01} \mathrm{Te}$
}

\author{
J. Trzmiel, E. PŁaczek-Popko, E. Zielony and Z. Gumienny \\ Institute of Physics, Wrocław University of Technology \\ Wybrzeże Wyspiańskiego 27, 50-370 Wrocław, Poland
}

\begin{abstract}
The dielectric response of gallium doped $\mathrm{Cd}_{0.99} \mathrm{Mn}_{0.01} \mathrm{Te}$ and CdTe alloys possessing DX centers was studied by impedance spectroscopy. Complex modulus and impedance spectroscopic plots were analyzed. Near ideal Debye response of CdTe:Ga was observed, whereas for $\mathrm{Cd}_{0.99} \mathrm{Mn}_{0.01} \mathrm{Te}$ : Ga samples non-Debye behavior was stated. Different relaxation responses may be related to various local atomic configurations in the vicinity of the DX centers in the studied materials.
\end{abstract}

PACS numbers: 71.55.Gs, 61.72.jj, 77.22.-d

\section{Introduction}

Wide-ranging studies of the relaxation phenomena have led to the conclusion that hardly ever experimentally observed relaxation responses are classical Debye ones. It appeared that various types of complex physical systems follow the non-exponential relaxation pattern. Generally, the non-exponential relaxation processes may be represented by the time domain KohlrauschWilliams-Watts $(\mathrm{KWW}) \Phi_{\mathrm{KWW}}(t)=\exp \left(-\left(\omega_{\mathrm{p}} t\right)^{\alpha}\right)$ and frequency domain the Havriliak-Negami (HN) $\Phi_{\mathrm{HN}}^{*}(\omega)=$ $\frac{1}{\left[1+\left(\mathrm{i} \omega / \omega_{\mathrm{p}}\right)^{\alpha}\right]^{\beta}}$ relaxation functions. In the above formulae $\omega_{\mathrm{p}}$ is characteristic material constant and $0<\alpha, \alpha \beta<1$. The parameters $\alpha=1$ and $\beta=1$ indicate the Debye-type relaxation. According to the probabilistic model of relaxation phenomenon [1] a material for which the near Debye macroscopic relaxation response is observed is purely deterministic. It means that each defect in the investigated sample possesses the same properties and defects relaxation rates are distributed according to Dirac- $\delta$ distribution. If the $\mathrm{HN}$ or KWW response is observed, it is a sign of different individual relaxation properties of the defects. In this case the distribution of defects relaxation rates is a heavy-tailed one.

It was reported in Ref. [2] that in the time domain persistent photoconductivity build-ups for gallium doped $\mathrm{Cd}_{1-x} \mathrm{Mn}_{x}$ Te mixed crystals follow the KWW relaxation pattern, whereas gallium doped CdTe samples exhibit double-exponential behavior. The non-exponentiality of photoconductivity transients in $\mathrm{Cd}_{1-x} \mathrm{Mn}_{x} \mathrm{Te}$ and $\mathrm{CdTe}$ results from the presence of deep metastable defects the so-called DX centers which are related to gallium (Ga) atoms. It was found that depending on the material the DX centers relaxation rates possess various relaxation rate distributions.

The objective of this paper is to analyze the dielectric response of these materials to bring into lime- light differences in frequency domain relaxation responses of $\mathrm{Cd}_{0.99} \mathrm{Mn}_{0.01} \mathrm{Te}: \mathrm{Ga}$ and CdTe:Ga. Gold Schottky junctions $\mathrm{Au}-\mathrm{Cd}_{1-x} \mathrm{Mn}_{x} \mathrm{Te}: \mathrm{Ga}$ and $\mathrm{Au}-\mathrm{CdTe}: \mathrm{Ga}$ were formed and impedance spectroscopy was applied to investigate the dielectric properties of samples.

\section{Experiment}

The samples of gallium doped $\mathrm{CdTe}$ and $\mathrm{Cd}_{0.99} \mathrm{Mn}_{0.01} \mathrm{Te}$ were processed by the Bridgman method. The room temperature, net donor concentration was found to be in the order of $10^{17} \mathrm{~cm}^{-3}$ in the CdTe:Ga and in the order of $10^{15} \mathrm{~cm}^{-3}$ in the $\mathrm{Cd}_{0.99} \mathrm{Mn}_{0.01} \mathrm{Te}: \mathrm{Ga}$ samples. Gold Schottky contacts were thermally evaporated on the front side of the samples.

The dielectric responses were measured using Novocontrol impedance analyzer. The ac probe signal amplitude equal to $10 \mathrm{mV}$ was applied. Measurements were performed at zero bias, in the frequency range from $0.2 \mathrm{~Hz}$ to $3 \mathrm{MHz}$, at temperatures in the range from $77 \mathrm{~K}$ to $300 \mathrm{~K}$.

\section{Result and discussion}

Frequently, to study dielectric characteristic of a sample frequency-dependent complex dielectric modulus $M^{*}(\omega)$ and impedance $Z^{*}(\omega)$ are considered and a simple parallel RC circuit representation of a Schottky junction is used. For a simple parallel RC circuit complex impedance and modulus are interrelated: $M^{*}(\omega)=M^{\prime}+\mathrm{i} M^{\prime \prime}=\mathrm{i} \omega C_{0} Z^{*}(\omega) . \quad C_{0}$ denotes the vacuum capacitance of the sample holder and electrode arrangement. Within this interpretation the imaginary part of modulus peak is scaled according to capacitance, the impedance peak - according to resistance. The parallel RC element results in a peak in spectroscopic plots of imaginary components $M^{\prime \prime}$ and $Z^{\prime \prime}$ versus frequency [3]. 

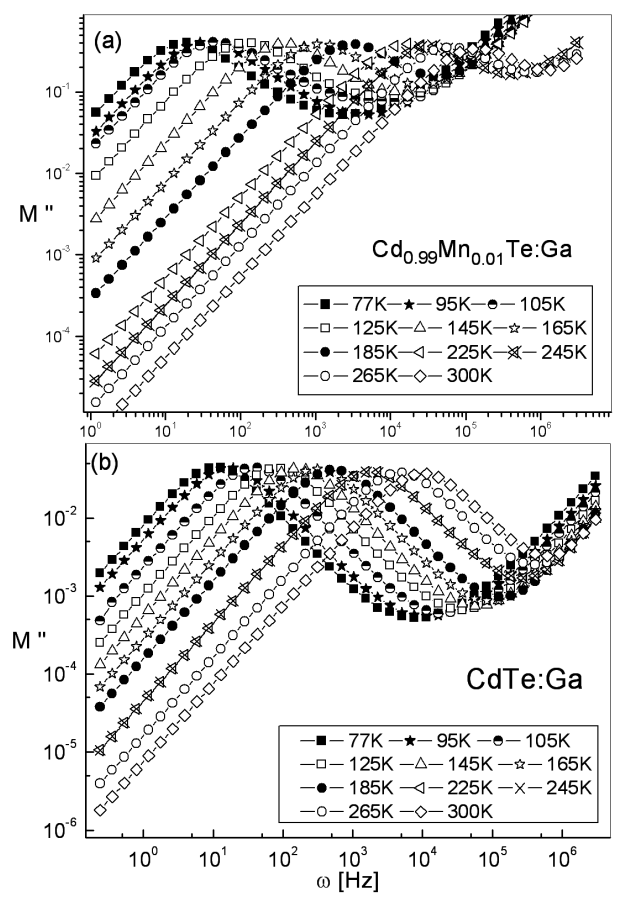

Fig. 1. Double-logarithmic scale plots of imaginary modulus versus frequency taken at different temperatures for $\mathrm{Cd}_{0.99} \mathrm{Mn}_{0.01} \mathrm{Te}: \mathrm{Ga}$ and CdTe:Ga.
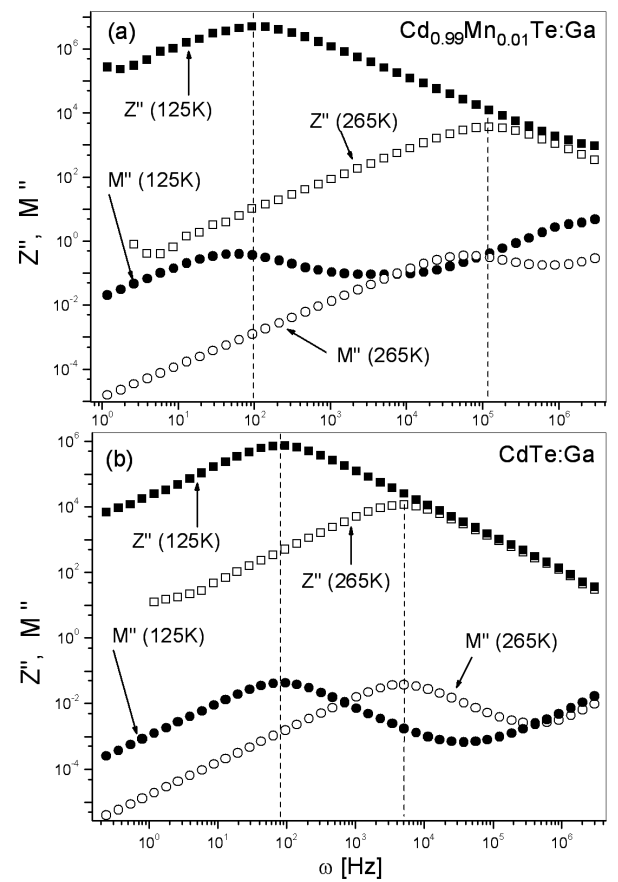

Fig. 2. Double-logarithmic imaginary modulus-impedance versus frequency plots measured at two different temperatures.

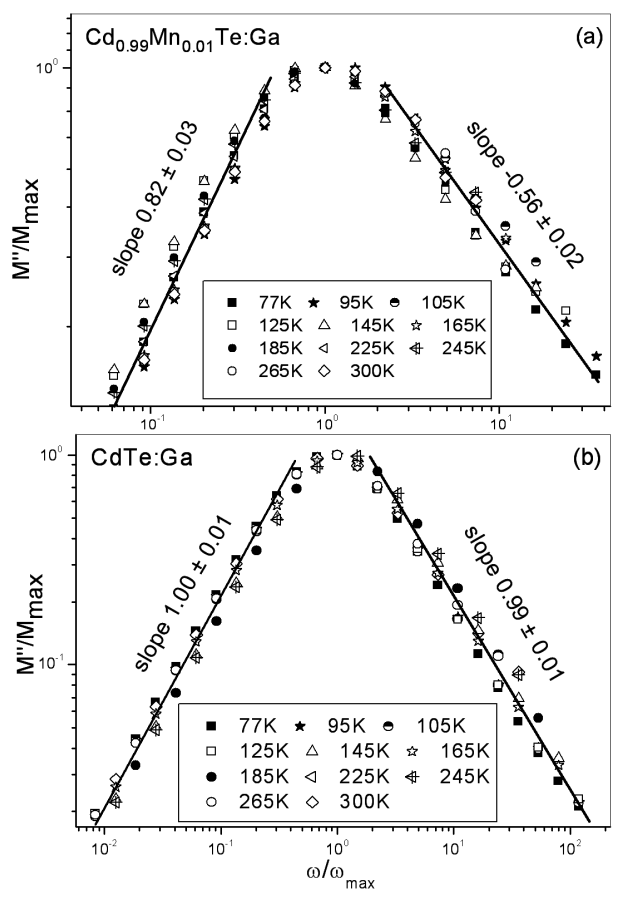

Fig. 3. Normalized imaginary modulus data for investigated temperature range (log-log scale). Data correspond to those given in Fig. 1.

In Fig. 1 the imaginary component of the modulus $M^{\prime \prime}$ as a function of frequency of the probing ac voltage at different temperatures is depicted for both $\mathrm{Cd}_{0.99} \mathrm{Mn}_{0.01} \mathrm{Te}: \mathrm{Ga}$ and CdTe:Ga. Regardless of sample, the modulus exhibits a single narrow peak with the low frequency edge shifted toward higher frequencies with increasing temperature. The observed single peak on $M^{\prime \prime}$ vs. frequency plots indicates the presence of a trap level within the depletion region of the considered Schottky junctions which dominates in the relaxation processes. The dominant deep trap in $\mathrm{Cd}_{1-x} \mathrm{Mn}_{x}$ Te:Ga as well as in CdTe:Ga is the metastable DX center, related to gallium [4].

In Fig. 2 the modulus-impedance versus frequency plots are shown. For $\mathrm{Cd}_{0.99} \mathrm{Mn}_{0.01} \mathrm{Te}$ :Ga the maximum of modulus and impedance corresponding to the same temperature are shifted, whereas for CdTe:Ga they peak at the same frequency irrespective of temperature. This observation implies a Debye type of relaxation for CdTe:Ga samples and a non-Debye for $\mathrm{Cd}_{0.99} \mathrm{Mn}_{0.01} \mathrm{Te}$ Ga.

In order to verify this supposition normalized imaginary part of the modulus presented in Fig. 3 has been analyzed. The normalization results in a single master curve, with almost temperature-independent peak amplitude. However, for CdTe:Ga sample, within the fitting error range, both branches of the peak possess slope coefficient close to 1 , pointing toward the near ideal Debye behavior. The dielectric response of $\mathrm{Cd}_{0.99} \mathrm{Mn}_{0.01} \mathrm{Te} \mathrm{Ga}$ does not follow the idealized Debye law, for both slopes are different from 1 . At this stage of the preliminary anal- 
ysis it is difficult to determine the formal classification of dielectric response of $\mathrm{Cd}_{0.99} \mathrm{Mn}_{0.01}$ Te: Ga mixed crystals but obtained results show that the HN type of response is the most probable. The obtained results strengthen our earlier observations based on the time domain relaxation measurements of photoconductivity build-ups. It has been found that for $\mathrm{Cd}_{0.99} \mathrm{Mn}_{0.01} \mathrm{Te}$ :Ga the time domain photoconductivity transients are properly described exclusively by the KWW relaxation pattern whereas the CdTe samples exhibit double-exponential photoconductivity response. It may be concluded that the stretched-exponentiality of the time-domain relaxation as well as the non-Debye pattern of the frequency-domain responses observed for the $\mathrm{Cd}_{0.99} \mathrm{Mn}_{0.01} \mathrm{Te}: \mathrm{Ga}$ material have its origin in a various atom configuration in the vicinity of the DX center in this alloy. This leads to the heavy-tailed distribution of the DX centers relaxation rates.

\section{Conclusions}

Relaxation properties of $\mathrm{Cd}_{0.99} \mathrm{Mn}_{0.01} \mathrm{Te} \mathrm{Ga}$ and CdTe:Ga samples were investigated by impedance spectroscopy in the frequency range from $0.2 \mathrm{~Hz}$ to $3 \mathrm{MHz}$, at temperatures in the range from $77 \mathrm{~K}$ to $300 \mathrm{~K}$. For both the materials one dominant bulk trap, presumably related to gallium dopant was detected in the complex modulus-impedance measurements. It was found, however, that the materials reveal various relaxation patterns - $\mathrm{Cd}_{0.99} \mathrm{Mn}_{0.01} \mathrm{Te}: \mathrm{Ga}$ a non-Debye one, CdTe:Ga - a near ideal Debye type. Differences in observed relaxation responses result from a variety of statistical properties of the DX centers in the investigated samples, which are related to different local atomic configuration in the vicinity of the defects.

\section{Acknowledgments}

Authors are grateful to Dr. J. Szatkowski for Schottky contacts preparation. The work has been financially supported by the Ministry of Science and Higher Education project No. N N202 091133.

\section{References}

[1] A. Jurlewicz, K. Weron, J. Non-Cryst. Solids 305, 112 (2002).

[2] J. Trzmiel, E. Płaczek-Popko, K. Weron, J. Szatkowski, E. Wojtyna, Acta Phys. Pol. A 114, 1417 (2008).

[3] A.K. Jonscher, Dielectric Relaxation in Solids, Chelsea Dielectric Press, London 1983.

[4] J. Szatkowski, E. Płaczek-Popko, K. Sierański, J. Fijałkowski, A. Hajdusianek, B. Bieg, Mater. Sci. Forum 258-263, 1413 (1997). 